\title{
Prélèvements nasopharyngés initialement négatifs chez un homme de 76 ans infecté par le SRAS-CoV-2
}

\author{
Divjot S. Kumar MD, Siobhán B. O’Neill MD PhD, James C. Johnston MD MPH, Jennifer M. Grant MD, \\ David D. Sweet MD
}

- Citation : CMAJ 2020 May 19;192:E546-9. doi: 10.1503/cmaj.200641-f; diffusion hâtive le 24 avril 2020

Voir la version anglaise de l'article ici : www.cmaj.ca/lookup/doi/10.1503/cmaj.200641

$\mathbf{U}$ n homme de 76 ans s'est présenté dans un centre hospitalier de soins quaternaires en raison de symptômes respiratoires, de fatigue et d'un épisode d'évanouissement dans les 6 jours précédents. Le patient présentait une toux sèche post-infectieuse en voie de résolution à la suite d'une infection des voies respiratoires supérieures survenue 3 mois avant la consultation. L'homme était atteint d'hypertension, d'une gastrite, de diabète et d'une sciatique, il avait des antécédents de tabagisme évalués à 30 paquets-année, mais pas d'antécédents d'utilisation de drogues à des fins récréatives. Il prenait de la metformine et de la rosuvastatine. L'homme était professeur et prenait des cours de langue.

Au début mars, le patient a été informé d'une possible exposition à la maladie à coronavirus 2019 (COVID-19). Une semaine après cette exposition, il a subi un prélèvement nasopharyngé en clinique externe pour dépistage du SRAS-CoV-2 (coronavirus du syndrome respiratoire aigu sévère 2) après 2 jours d'une toux qui s'aggravait. Il a obtenu un résultat négatif pour les gènes encodant l'enveloppe et l'ARN-polymérase dépendante de l'ARN, qui sont les cibles courantes des tests de réaction en chaîne par polymérase (PCR) pour le SRAS-CoV-2. Pendant les 4 jours suivants, le patient a présenté une aggravation de sa toux sèche, de la fatigue, une dyspnée à l'effort, de la fièvre, une baisse de l'appétit et de la diarrhée. Il a consulté à l'urgence 4 jours après son premier prélèvement de dépistage du SRAS-CoV-2 (11 jours après l'exposition potentielle).

Le patient a été admis à l'hôpital, avec des mesures de précaution contre la transmission par gouttelettes ou contact. Ses signes vitaux initiaux comprenaient une température corporelle de $38,6^{\circ} \mathrm{C}$, une tension artérielle de $98 / 55 \mathrm{~mm} \mathrm{Hg}$ avec baisse orthostatique, une fréquence cardiaque de 94 battements/ minute et une fréquence respiratoire de 18 respirations/minute, avec saturation en oxygène de $96 \%$ à l'air ambiant. L'examen physique a révélé un affaissement des veines du cou. II présentait des crépitations bibasilaires légères à l'inspiration. Les analyses sanguines ont montré une lymphopénie, mais les électrolytes et les épreuves de fonction hépatique et rénale étaient normales.

\section{POINTS CLÉS}

- La sensibilité des tests de RT-PCR (réaction en chaîne par polymérase après transcription inverse) en temps réel pour les prélèvements nasopharyngés ou oropharyngés dans les cas d'infection par le coronavirus du syndrome respiratoire aigu sévère (SRAS-CoV-2) dépend de la technique d'écouvillonnage et du moment où il est effectué au cours de la maladie.

- Des études ont montré des résultats négatifs aux tests nasopharyngés pour le SRAS-CoV-2 chez des patients présentant une maladie à coronavirus 2019 selon les symptômes cliniques qui se sont par la suite révélés positifs.

- La possibilité d'une acquisition et d'une transmission nosocomiales du SRAS-CoV-2 par des travailleurs de la santé asymptomatiques justifie le recours aux précautions universelles pour soigner les patients.

La radiographie pulmonaire a montré une opacification aux contours flous à la base droite.

On a commencé un traitement contre la pneumonie par cristalloïdes intraveineux, avec ceftriaxone et azithromycine. Les cultures de sang et de selles effectuées à l'admission étaient négatives; les virus de la grippe $A$ et $B$, de même que le virus respiratoire syncytial étaient absents; et un deuxième prélèvement nasopharyngé pour déceler le SRAS-CoV-2 à l'admission a aussi produit des résultats négatifs.

Trois jours après son admission, le patient est devenu hypoxémique et avait besoin de $2 \mathrm{~L}$ d'oxygène par minute. Une nouvelle radiographie pulmonaire a révélé de nouvelles opacités bilatérales aux contours flous. Les résultats d'un panel viral élargi, de la recherche d'antigènes urinaires contre la légionellose et d'un troisième prélèvement nasopharyngé pour le SRASCoV-2 se sont révélés négatifs. Nous avons suspendu les précautions contre la transmission par gouttelettes ou contact au jour 3 d'hospitalisation. Une tomodensitométrie (TDM) thoracique a révélé la présence d'une hyperdensité bilatérale en verre dépoli et des lignes septales (figure 1) concordant avec une infection bactérienne ou virale atypique; le diagnostic différentiel incluait 
un œdème, une hémorragie, une réaction médicamenteuse et une maladie des tissus conjonctifs.

Au jour 4 d'hospitalisation, l'hypoxémie du patient s'est aggravée. Il est passé à $5 \mathrm{~L}$ d'oxygène par minute. Nous avons consulté le département de pneumologie. Compte tenu de la progression de ses symptômes, de la lymphopénie persistante et d'une exposition potentielle à un étudiant porteur de la COVID19 , on nous a conseillé de réinstaurer les précautions contre la
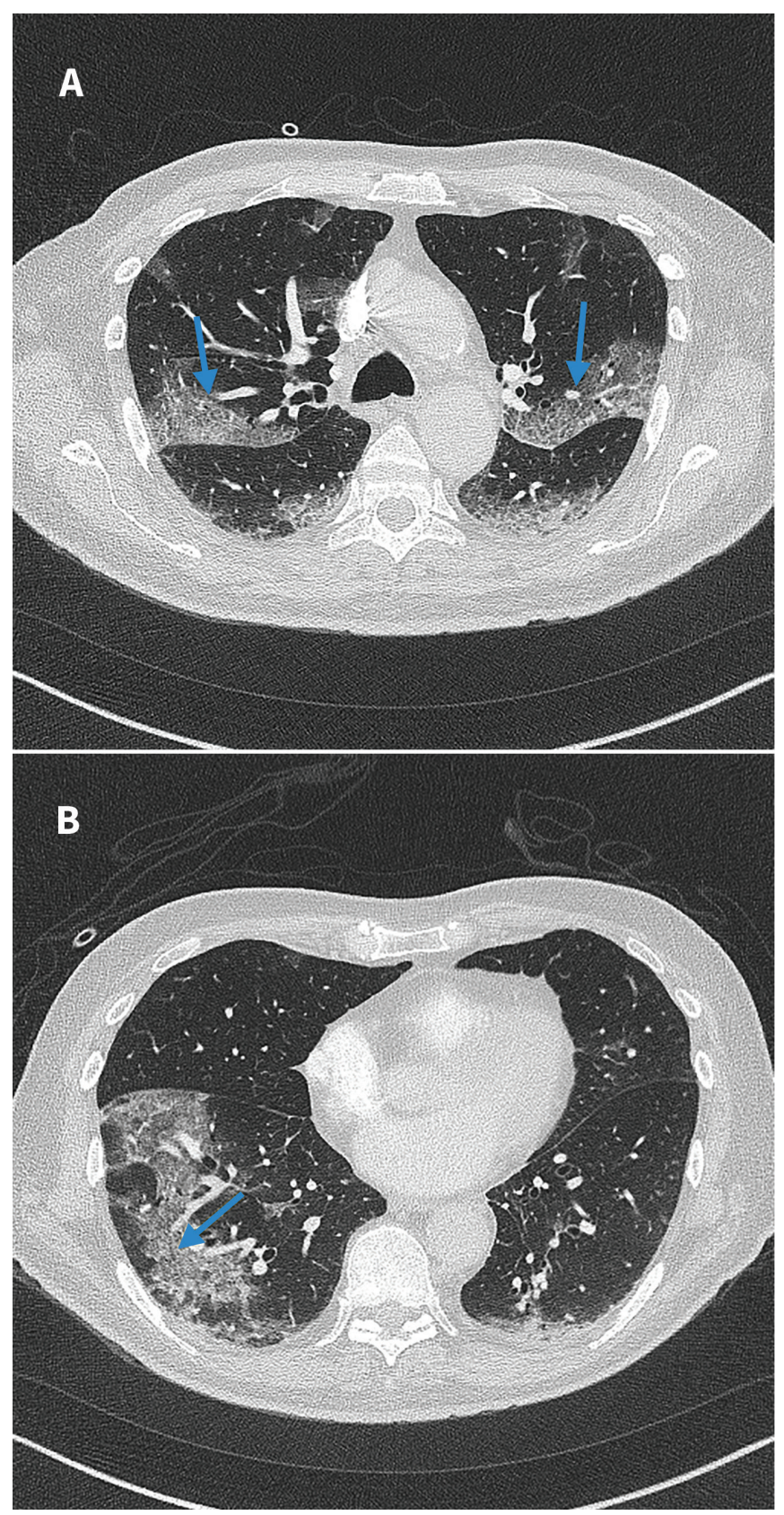

Figure 1 : Clichés de TDM thoracique (A) et (B) (pris au jour 3 d'hospitalisation) chez un homme de 76 ans porteur de la maladie à coronavirus 2019 (COVID-19) et ayant eu des résultats négatifs aux prélèvements nasopharyngés. On observe une hyperdensité périphérique bilatérale en verre dépoli avec zones de lignes septales constituant une mosaïque (flèches bleues). Il s'agit d'un aspect typique de la COVID-19, selon l'énoncé consensuel des experts de la Radiological Society of North America ${ }^{1}$. transmission par gouttelettes ou contact ${ }^{1}$. Nous avons changé l'antibiothérapie en cours pour des antibiotiques à plus large spectre (pipéracilline/tazobactam et vancomycine). Nous avons aussi instauré un traitement empirique par méthylprednisolone (125 mg par voie intraveineuse 1 fois par jour) pour une possible pneumonie organisée. Les cultures d'expectorations et un quatrième prélèvement nasopharyngé pour le dépistage du SRAS-CoV-2 réalisé au jour 4 d'hospitalisation se sont révélés négatifs. Nous avons transféré le patient à l'unité des soins intensifs (USI), où on l'a soigné dans une chambre à pression négative. Un traitement diurétique a été tenté, et le patient a été placé sous fraction inspirée en oxygène $\left(\mathrm{FiO}_{2}\right)$ de $60 \%$ par canule nasale à haut débit.

Au jour 5 , il a fallu augmenter la $\mathrm{FiO}_{2}$ à $90 \%$. Il a été intubé, ventilé et soumis à un protocole de protection pulmonaire. La posologie de la méthylprednisolone a été portée à $125 \mathrm{mg}$ par voie intraveineuse toutes les 6 heures. Les résultats des tests pour la maladie des tissus conjonctifs, la maladie des anticorps anti-membrane basale glomérulaire, l'hépatite et le VIH étaient négatifs.

Au jour 6, la bronchoscopie a révélé la présence de sang dans les liquides de lavage bronchoalvéolaire séquentiels. Les tests de PCR après transcription inverse (RT-PCR) sur les échantillons de lavage et de brossage bronchoalvéolaires ont donné des résultats positifs pour le SRAS-CoV-2. Au jour 7 d'hospitalisation, les résultats des prélèvements nasopharyngés et des sécrétions trachéales étaient positifs pour le SRAS-CoV-2. Le patient a reçu un traitement de soutien et a été extubé au jour 10. Nous avons réduit sa prednisone à $50 \mathrm{mg} / \mathrm{j}$, puis l'avons diminuée par paliers de $5 \mathrm{mg}$ tous les 3 jours. Une nouvelle TDM au jour 15 (figure 2) a révélé une embolie pulmonaire segmentaire et des infiltrats pulmonaires en évolution concordant avec une pneumonie organisée. Nous avons commencé un traitement anticoagulant et le patient a reçu son congé 19 jours après son admission.

\section{Discussion}

Il faut à tout prix reconnaître sans tarder les patients porteurs de la COVID-19 pour pouvoir mettre en place les mesures d'isolement et de traitement appropriées. Notre cas illustre certains des défis diagnostiques entourant la COVID-19 et leurs conséquences pour la prise en charge, y compris la prévention de la transmission nosocomiale.

Même si les tests de RT-PCR sont la norme actuelle pour le dépistage du SRAS-CoV-2 et le diagnostic de la COVID-19, selon des rapports, la sensibilité des prélèvements nasopharyngés est variable, allant de $42 \%$ à $71 \%{ }^{1,2}$. Dans une récente série de cas présentant une forte probabilité de COVID-19 d'après le tableau clinique et l'imagerie, seulement 9 patients sur 19 (47\%) avaient des tests oropharyngés positifs par RT-PCR ${ }^{3}$. La sensibilité de l'écouvillonnage pour le SRAS-CoV-2 dépendrait de la technique de prélèvement et du moment où il est effectué durant le cours de la maladie ${ }^{2-4}$. En outre, les signes à la TDM thoracique pourraient précéder les résultats positifs des tests de RT-PCR ${ }^{1,2}$. Le cas récent d'un Thaïlandais de 28 ans présentant une toux a fait état de 2 résultats de prélèvements nasopharyngés négatifs au jour 5 

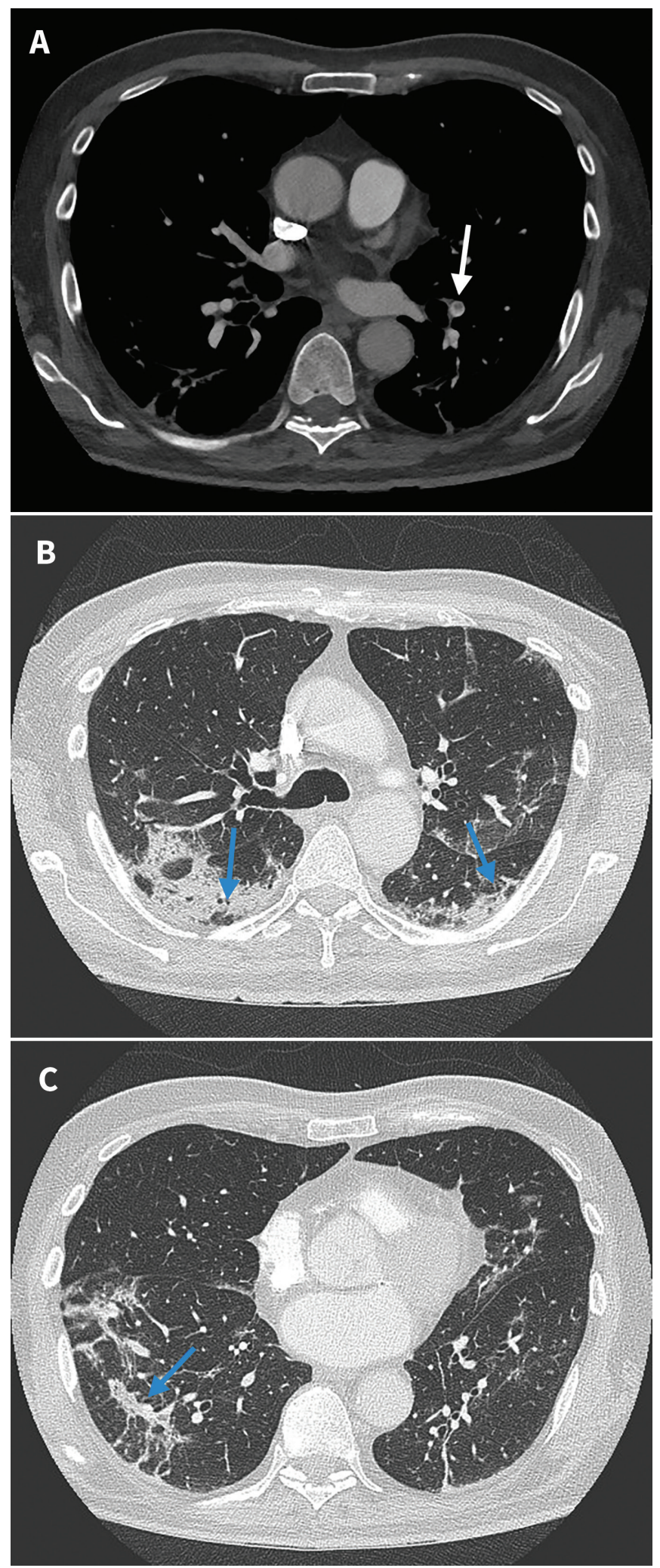

Figure 2 : Clichés de TDM thoracique pris au jour 15 d'hospitalisation. (A) Embolie pulmonaire segmentaire non occlusive au lobe inférieur gauche (flèche blanche). (B) et (C) Amélioration de l'aspect en verre dépoli et épaississement septal; consolidation péribronchovasculaire et périlobulaire périphérique bilatérale avec légère perte de volume et distorsion parenchymateuse concordant avec l'évolution type d'une pneumonie organisée en cours d'évolution (flèches bleues). de son hospitalisation, alors que la bronchoscopie subséquente effectuée au jour 8 par lavage bronchoalvéolaire a donné un résultat positif pour le SRAS-CoV-2 par RT-PCR . De telles différences pourraient s'expliquer par l'incubation prolongée qui affecte la sensibilité des prélèvements pharyngés. Une étude in vitro réalisée durant l'épidémie de SRAS a montré que le récepteur fonctionnel du SRAS-CoV était l'enzyme de conversion de l'angiotensine 2 (ECA2) ${ }^{5}$. L'expression minimale de l'ECA2 au niveau du pharynx postérieur, comparativement aux voies respiratoires inférieures, pourrait expliquer les différences de charge virale selon le site anatomique, et réduirait la sensibilité des prélèvements nasopharyngés comparativement aux échantillons provenant des voies respiratoires bases ${ }^{4,5}$. La similarité génomique entre le SRAS-CoV et le SRAS-CoV-2 a mené certains chercheurs à formuler une hypothèse selon laquelle des mécanismes similaires pourraient s'appliquer au cycle de réplication actuel du SRAS-CoV- $2^{6}$.

L'importance de comprendre les tests diagnostiques en fonction du stade clinique de la COVID-19 est encore soulignée par une récente étude qui a évalué les charges virales dans différents sites anatomiques et a révélé que les concentrations d'ARN du SRAS-CoV-2 dans les prélèvements pharyngés étaient déjà en baisse au moment de la première consultation clinique, tandis que les concentrations d'ARN viral dans les expectorations diminuaient plus lentement ${ }^{7}$. Les auteurs de cette étude ont noté une sécrétion virale pharyngée active dans la maladie légère, avec atteinte croissante des voies respiratoires basses plus tard au cours de la maladie. Selon une récente ligne directrice, l'utilisation de prélèvements provenant des voies respiratoires basses, si possible, plutôt que de prélèvements nasopharyngés, pourrait améliorer la précision des tests de RT-PCR. Malheureusement, il n'est pas facile d'obtenir des échantillons des voies respiratoires basses chez tous les patients que l'on soupçonne d'être atteints de la COVID-19. La bronchoscopie permet d'accéder aux voies respiratoires basses, mais il s'agit d'une intervention qui génère des aérosols et peut exposer les travailleurs de la santé à un risque de contamination.

Il est possible que notre patient ait été exposé au SRAS-CoV-2 à l'hôpital. Cela suppose qu'il aurait présenté une insuffisance respiratoire hypoxémique secondaire à une pneumonie organisée ou à une alvéolite due à autre chose que le SRAS-CoV-2. Ses prélèvements nasopharyngés initiaux à l'hôpital peuvent avoir été réellement négatifs, avec des résultats de bronchoscopie subséquents reflétant une acquisition nosocomiale du SRASCoV-2. De plus, la période d'incubation de la COVID-19 est de 2 à 11 jours (en moyenne 5 jours), ce qui correspond à une exposition possible au SRAS-CoV-2 durant la brève période où il n'a pas été isolé. La possibilité d'une propagation nosocomiale du SRAS-CoV-2 est de plus en plus reconnue; même si on ne suggère pas de procéder à un dépistage à grande échelle des travailleurs de la santé asymptomatiques pour l'instant, la transmission du SRAS-CoV-2 par des porteurs asymptomatiques a été notée ${ }^{10}$. Pour réduire le risque de transmission, l'Hôpital général de Vancouver a établi une politique de port de masque et de protection oculaire universelle pour tous les travailleurs de la santé en contact direct avec des patients. Compte tenu de la possibilité d'une 
transmission nosocomiale, il faut veiller à une stricte observance des précautions et à une hygiène optimale de la part des travailleurs de la santé asymptomatiques.

L'utilisation systématique de la corticothérapie pour le traitement de la COVID-19 n'est actuellement pas recommandée, à moins d'autres indications ${ }^{8}$, chez les patients atteints de la COVID-19 et du syndrome de détresse respiratoire aiguë. Toutefois, l'utilisation des corticostéroïdes reste controversée. Les lignes directrices de la Surviving Sepsis Campaign contiennent une recommandation faible en faveur de la corticothérapie chez les patients atteints de la COVID-19 et du syndrome de détresse respiratoire aiguë ${ }^{8}$. Dans le cas présent, il est possible que l'administration des corticostéroïdes à forte dose ait accéléré la réplication virale et l'obtention d'un résultat positif pour le SRASCoV-2 3 jours plus tard. Cette question a été explorée lors de la précédente éclosion de SRAS, où l'utilisation hâtive des corticostéroïdes a semblé entraîner une augmentation des charges virales plasmatiques et un retard de l'élimination virale ${ }^{11}$. Compte tenu du rétablissement rapide de notre patient à l'USI alors qu'il recevait une corticothérapie, nous avons maintenu les corticostéroïdes avec sevrage rapide afin d'abréger la période d'élimination virale.

\section{Conclusion}

Les prélèvements nasopharyngés pour le dépistage du SRASCoV-2 par RT-PCR devraient être interprétés en tenant compte des signes et symptômes cliniques. S'il demeure un soupçon clinique de COVID-19 après un prélèvement nasopharyngé initialement négatif, nous enjoignons les cliniciens à maintenir l'isolement pour prévenir les infections nosocomiales.

\section{Références}

1. Simpson S, Kay FU, Abbara S, et al. Radiological Society of North America Expert Consensus Statement on Reporting Chest CT Findings Related to COVID-19. Endorsed by the Society of Thoracic Radiology, the American College of Radiology, and RSNA. Radiology: Cardiothoracic Imaging 2020 Mar. 25;2:e200152. doi: 10.1148/ryct.2020200152.

2. Ai T, Yang Z, Hou H, et al. Correlation of chest CT and RT-PCR testing in coronavirus disease 2019 (COVID-19) in China: a report of 1014 cases. Radiology 2020 Feb. 26 [Cyberpublication avant impression]. 200642. doi: 10.1148/radiol .2020200642
3. Xie C, Jiang L, Huang G, et al. Comparison of different samples for 2019 novel coronavirus detection by nucleic acid amplification tests. Int J Infect Dis 2020; 93:264-7.

4. Winichakoon P, Chaiwarith R, Liwsrisakun C, et al. Negative nasopharyngeal and oropharyngeal swab does not rule put COVID-19. J Clin Microbiol 2020 Feb. 26 [Cyberpublication avant impression]. pii: JCM.00297-20. doi: 10.1128/ JCM.00297-20.

5. Li W, Moore MJ, Vasilieva N, et al. Angiotensin-converting enzyme 2 is a functional receptor for the SARS coronavirus. Nature 2003;426:450-4.

6. Vaduganathan M, Vardeny $\mathrm{O}$, Michel $\mathrm{T}$, et al. Renin-angiotensin-aldosterone system inhibitors in patients with COVID-19. NEJM 2020 Mar. 30 [Cyberpublication avant impression]. doi: 10.1056/NEJMsr2005760.

7. Woelfel R, Corman VM, Guggemos W, et al. Clinical presentation and virological assessment of hospitalized cases of coronavirus disease 2019 in a travelassociated transmission cluster. medRxiv 2020 Mar 8. doi: 10.1101/2020.03.05 20030502 .

8. Alhazzani W, Møller MH, Arabi YM, et al. Surviving sepsis campaign: guidelines on the management of critically ill adults with coronavirus disease 2019 (COVID-19). Intensive Care Med 2020 Mar. 28 [Cyberpublication avant impression]. doi: 10.1007/s00134-020-06022-5.

9. Backer JA, Klinkenberg D, Wallinga J. Incubation period of 2019 novel coronavirus (2019-nCoV) infections among travellers from Wuhan, China, 20-28 January 2020 [communication rapide]. Euro Surveill 2020 Feb. 6 [Cyberpublication avant impression];25. doi: 10.2807/1560-7917.ES.2020.25.5.2000062.

10. Klompas M, Morris CA, Sinclair J, et al. Universal masking in hospitals in the COVID-19 era. N Engl J Med 2020 Apr. 1 [Cyberpublication avant impression]. doi: 10.1056/NEJMp2006372.

11. Lee N, Allen Chan KC, Hui DS, et al. Effects of early corticosteroid treatment on plasma SARS-associated coronavirus RNA concentrations in adult patients. J Clin Virol 2004;31:304-9.

Intérêts concurrents : Aucun déclaré.

Cet article a été révisé par des pairs.

Les auteurs ont obtenu le consentement du patient.

Affiliations : Divisions de pneumologie (Kumar, Johnston), d'infectiologie (Grant) et de médecine de soins intensifs (Sweetuce), Département de radiologie (O’Neill), Faculté de médecine, Université de la Colombie-Britannique, Vancouver, C.-B.

Collaborateurs : Divjot Kumar a rédigé la première version du manuscrit. Tous les auteurs ont contribué à la conception du travail et ont révisé de façon critique le contenu intellectuel important; ils ont donné leur approbation finale pour la version destinée à être publiée et assument l'entière responsabilité de tous les aspects du travail.

Remerciements : Les auteurs remercient les $D^{\text {rs }}$ Tharwat Fera et Robert Reynolds pour leur aide lors de la prise en charge du cas.

Correspondance : Divjot Kumar, kumardivjot@gmail.com 